\title{
Interaction of ultra-energetic cosmic neutrinos with a thermal gas of relic neutrinos
}

\author{
Véronique Van Elewyck* \\ Instituto de Ciencias Nucleares, Universidad Nacional Autónoma de México \\ Mexico City, Mexico \\ E-mail: vero@nucleares.unam.mx
}

\section{J. C. D'Olivo, L. Nellen and S. Sahu}

Instituto de Ciencias Nucleares, Universidad Nacional Autónoma de México

Mexico City, Mexico

\begin{abstract}
We use the formalism of finite-temperature field theory to study the interactions of ultra-high energy (UHE) cosmic neutrinos with the thermal background of relic neutrinos. From the imaginary part of the neutrino self-energy, calculated in terms of the $\mathrm{Z}$ boson propagator near the resonance, we derive general expressions for the UHE neutrino transmission probability. This allows us to take into account the thermal effects introduced by the momentum distribution of the relic neutrinos. We compare our results with the approximate expressions existing in the literature and discuss the influence of thermal effects on the absorption dips in the context of realistic UHE neutrino fluxes and favoured neutrino mass schemes.
\end{abstract}

International Europhysics Conference on High Energy Physics

July 21st - 27th 2005

Lisboa, Portugal

${ }^{*}$ Speaker. 


\section{Introduction}

The interaction of cosmic neutrinos at ultra-high energies (UHEv) with the cosmological background of relic (anti)neutrinos $(\mathrm{C} v \mathrm{~B})$ has been proposed as a way of observing the $\mathrm{C} v \mathrm{~B}$, and a method to perform relic neutrino spectroscopy [1]. Provided adequate sensitivity and energy resolution of the detectors, the observation in the UHE $v$ flux of absorption lines associated with the resonant production of a $Z$ boson $(v \bar{v} \rightarrow Z \rightarrow f \bar{f})$ could indeed allow an indirect determination of the absolute neutrino masses. The shape and depth of these absorption dips may also reflect features of the distribution of UHE $v$ sources and of their emission spectrum [2]. Most of the work in the literature describe the UHE $v-\mathrm{C} v \mathrm{~B}$ interactions assuming that relic neutrinos are at rest. However, effects of thermal motion in the $\mathrm{C} v \mathrm{~B}$ (whose present temperature is $\approx 1.69 \times 10^{-4} \mathrm{eV}$ ) become relevant as soon as the momentum of the relic neutrinos gets comparable to their mass, and even before. To take this effect into account, we compute the dominant (resonant) contribution to the neutrino damping using the real-time formalism of finite-temperature field theory (FTFT), and investigate the modifications in the UHE $v$ transmission probability due to thermal effects [5].

\section{Damping of UHE $v$ across the $\mathbf{C} v \mathbf{B}$}

For an UHE $v$ with four-momentum $k^{\mu}=\left(\mathscr{E}_{K}, \vec{K}\right)$ and mass $m_{v}$ travelling across the $\mathrm{C} v \mathrm{~B}$, the equation of motion reads $\left(\not k-m_{v}-\Sigma\right) \psi=0$, where the self-energy $\Sigma$ embodies the effects of the medium. The corresponding dispersion relation is given by $\mathscr{E}_{K}=\mathscr{E}_{r}(K)-i \gamma(K) / 2$. In our case, $\Sigma$ is determined from a FTFT one-loop calculation carried out in terms of the (vacuum) $\mathrm{Z}$ boson propagator and the thermal propagator of the relic neurinos. The last one depends on the functions $f_{V}(P)$ and $f_{\bar{v}}(P)$ which describe the momentum distributions of neutrinos (antineutrinos) in the thermal bath. These functions take the simple relativistic Fermi-Dirac form, $f_{v}(P)=f_{\bar{v}}(P)=1 /\left(e^{P / T_{v}}+1\right)$, where $T_{v}$ is the temperature of the $\mathrm{C} v \mathrm{~B}$ and we have neglected the chemical potential.

The damping factor $\gamma$ governs the propagation of the UHE $v$ across the background of relic neutrinos and is directly related to the imaginary part of the self-energy, $\Sigma_{i}$ [4]. In the approximation that the UHE $v$ are ultrarelativistic and we can neglect the background effects on their energy $\left(\mathscr{E}_{r}(K) \simeq K\right)$, the damping can be written as (see [5] for the detailed calculation)

$$
\gamma_{v \bar{v}}(K)=-\left.\frac{1}{K} \operatorname{Tr}\left(k \Sigma_{i}\right)\right|_{\mathscr{E}_{r}=K}=\int_{0}^{\infty} \frac{d P}{2 \pi^{2}} P^{2} f_{\bar{v}}(P) \sigma_{v \bar{v}}(P, K) .
$$

For $m_{v} \ll M_{Z}, K$ and neglecting terms of order $\Gamma_{Z}^{2} / M_{Z}^{2}$, we have

$$
\begin{aligned}
\sigma_{v \bar{v}}(P, K)= & \frac{2 \sqrt{2} G_{\mathrm{F}} \Gamma_{Z} M_{Z}}{2 K E_{p}}\left\{1+\frac{M_{Z}^{2}}{4 K P} \ln \left(\frac{4 K^{2}\left(E_{p}+P\right)^{2}-4 M_{Z}^{2} K\left(E_{p}+P\right)+M_{Z}^{4}}{4 K^{2}\left(E_{p}-P\right)^{2}-4 M_{Z}^{2} K\left(E_{p}-P\right)+M_{Z}^{4}}\right)\right. \\
& \left.+\frac{M_{Z}^{3}}{4 K P \Gamma_{Z}}\left[\arctan \left(\frac{2 K\left(E_{p}+P\right)-M_{Z}^{2}}{\Gamma M_{Z}}\right)-\arctan \left(\frac{2 K\left(E_{p}-P\right)-M_{Z}^{2}}{\Gamma M_{Z}}\right)\right]\right\},
\end{aligned}
$$

where $E_{p}=\sqrt{P^{2}+m_{v}^{2}}$ is the energy of the relic neutrino. Taking the limit of eq.(2.2) for $P \rightarrow 0$, one recovers the approximated cross-section used for relic neutrinos at rest, with the $\mathrm{Z}$ peak at the UHE $v$ "bare" resonance energy $K_{\text {res }}=M_{Z}^{2} /\left(2 m_{v}\right)$. However, this approximation breaks down for 

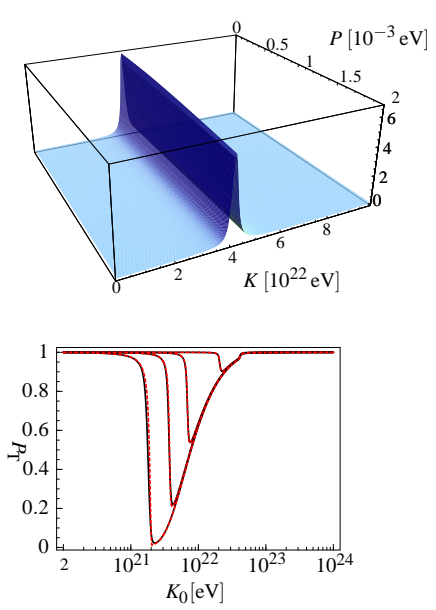
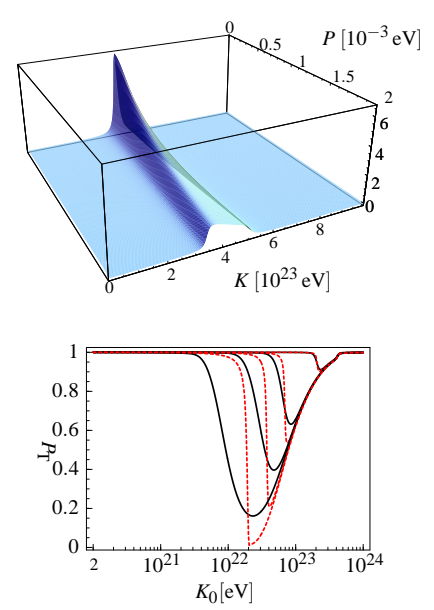
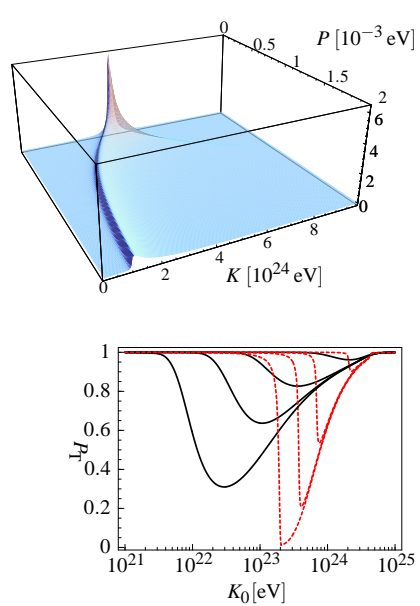

Figure 1: Top : cross-section $\sigma_{v \bar{v}}(P, K)$, in units of $10^{-31} \mathrm{~cm}^{2}$, as given by eq. (2.2), as a function of the energy of the incident neutrino, $K$, and of the relic neutrino momentum, $P$. From left to right, the panels correspond to a neutrino mass $10^{-1}, 10^{-2}$, and $10^{-3} \mathrm{eV}$. Bottom : Transmission probability $P_{\mathrm{T}}\left(K_{0}, z_{\mathrm{s}}\right)$ as a function of the UHE $v$ energy as detected on Earth, $K_{0}$, for a source located at redshifts $z_{\mathrm{s}}=1,5,10,20$ (from top to bottom in each panel) and for a neutrino mass $m_{v}=10^{-1}, 10^{-2}, 10^{-3} \mathrm{eV}$ (from left to right). The continued, black curves correspond to the full damping as given by eqs. (2.1) and (2.2), while the dotted (red) curves are for the approximation of relic neutrinos at rest.

small $m_{v}$ : Fig. 1 (top line) shows how the resonance peak in the $v \bar{v} \rightarrow Z$ cross-section broadens and shifts to lower UHE $v$ energies as $P$ increases. The transmission probability for an UHE $v$ emitted at a redshift $z_{s}$ to be detected on Earth with an energy $K_{0}$ is obtained by integrating the damping along the UHE $v$ path, taking into account that both the UHE $v$ energy and the $\mathrm{C} v \mathrm{~B}$ temperature are redshifted:

$$
P_{\mathrm{T}}\left(K_{0}, z_{\mathrm{s}}\right)=\exp \left[-\int_{0}^{z_{\mathrm{s}}} \frac{d z}{H(z)(1+z)} \gamma_{v \bar{v}}\left(K_{0}(1+z)\right)\right],
$$

where $H=H_{0} \sqrt{0.3(1+z)^{3}+0.7}$ is the Hubble factor. Fig. 1 (bottom line) shows that for $m_{v} / T_{v} \lesssim$ $10^{2}$ the absorption lines are also significantly broadened and shifted to lower energies, and that the effect increases with the distance travelled by the UHE $v$. This complicates the extraction of $m_{v}$ and $z_{s}$ from the start- and endpoint of the absorption dip, which, in the approximation of relic neutrinos at rest, were respectively located at $K_{0}=K_{\text {res }} /\left(1+z_{s}\right)$ and $K_{0}=K_{\text {res }}=M_{Z}^{2} /\left(2 m_{v}\right)$.

\section{Absorption lines in the UHE $v$ flux}

To investigate this effect in a realistic context, we applied our calculation to a flux of UHEv

$$
\mathscr{F}_{v}\left(K_{0}\right)=\frac{1}{4 \pi} \int_{0}^{\infty} \frac{d z}{H(z)} P_{\mathrm{T}}\left(K_{0}, z\right) \eta(z) J_{v}\left(K_{0}\right),
$$

assuming a distribution of sources $\eta(z)=\eta_{0}(1+z)^{n} \theta\left(z-z_{\min }\right) \theta\left(z_{\max }-z\right)$ with a common injection spectrum $J_{v}(K)=j_{v} K^{-\alpha} \theta\left(K_{\max }-K\right)$. The normalized flux then only depends on the difference of spectral indexes, $\alpha-n$, with typically $\alpha-n \approx 2$ for astrophysical (bottom-up) sources and $\alpha-n \approx 0$ for top-down processes [2]. For these two cases, we computed the normalized, all-flavour UHE $v$ spectrum assuming some mass patterns compatible with the currently favoured three-neutrinos mass schemes [3]. Fig. 2 shows how thermal broadening affects the superposition of absorption lines and globally modifies the shape and extension of the dip. 

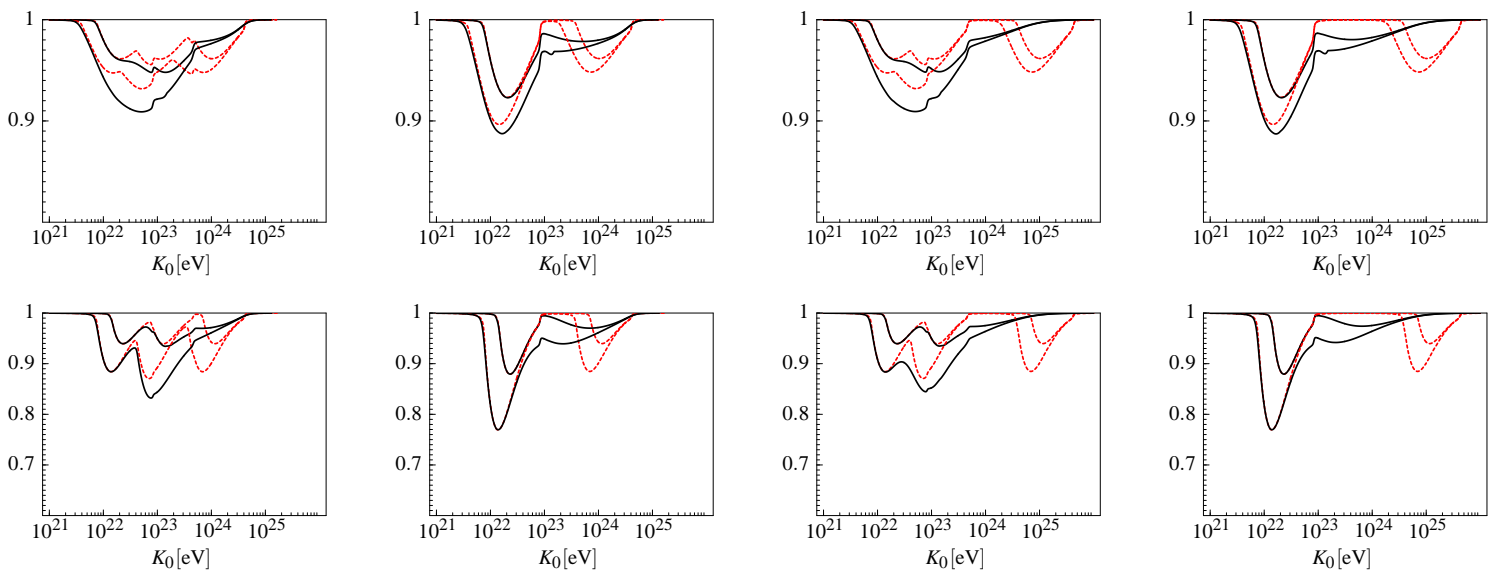

Figure 2: UHE $v$ flux in presence of damping, $\mathscr{F}_{v}$, normalized to the corresponding flux in absence of interactions. The top row is for $\alpha-n=0$ and $z_{s}=10,20$, and the bottom row is for $\alpha-n=2$ and $z=5,10$. From left to right, columns correspond to the following neutrino mass patterns: $\left\{10^{-3}, 9 \times 10^{-3}, 5 \times 10^{-2}\right\},\left\{10^{-3}, 5 \times 10^{-2}, 5 \times 10^{-2}\right\}$, $\left\{10^{-4}, 9 \times 10^{-3}, 5 \times 10^{-2}\right\},\left\{10^{-4}, 5 \times 10^{-2}, 5 \times 10^{-2}\right\}$ (all in eV). Colour code is as in fig. 1 .

\section{Conclusions}

From the exploration of the parameter space currently allowed by astrophysical and cosmological constraints, we see that thermal effects do affect the transmission properties of UHE $v$ across the $\mathrm{C} v \mathrm{~B}$ even in the regime of non-relativistic relic neutrinos. For most neutrino mass patterns, the extraction of the neutrino masses from the endpoint of the absorption lines is complicated by the broadening and merging of the dips, especially in normal hierarchical schemes (columns 1 and 3 in fig. 2) and for top-down-like injection spectra. Some information on the source distribution could still be provided by the onset energy and slope of the dip.

\section{Acknowledgements}

We would like to acknowledge support by CONACyT under grants 34868-E and 46999-F and by DGAPA-UNAM under grants PAPIIT IN116503, IN119405, and IN112105.

\section{References}

[1] T. J. Weiler, Phys. Rev. Lett. 49, 234 (1982) and Astrophys. J. 285, 495 (1984); E. Roulet, Phys. Rev. D 47, 5247 (1993); H. Pas and T. J. Weiler, Phys. Rev. D 63 (2001) 113015; D. Fargion, P. G. De Sanctis Lucentini, M. Grossi, M. De Santis, and B. Mele, Mem. Soc. Ast. It. 73 (2002) 848.

[2] B. Eberle, A. Ringwald, L. Song, and T. J. Weiler, Phys. Rev. D 70, 023007 (2004); G. Barenboim, O. Mena Requejo, and C. Quigg, Phys. Rev. D 71 (2005) 083002.

[3] J. F. Beacom and N. F. Bell, Phys. Rev. D 65 (2002) 113009.

[4] J. C. D’Olivo and J. F. Nieves, Phys. Rev. D 52 (1995) 2987.

[5] J. C. D’Olivo, L. Nellen, S. Sahu and V. Van Elewyck, arXiv:astro-ph/0507333, to appear in Astropart. Phys. 